\title{
Multiple bronchiolar stenoses in a patient with generalized airways obstruction
}

\author{
JOHN R. ESTERLY AND BRIAN E. HEARD \\ From the Postgraduate Medical School of London
}

In a previous article three cases of irreversible airways obstruction with minimal emphysema were described (Simpson, Heard, and Laws, 1963). Ventilatory function was grossly impaired, but special methods for emphysema applied to the lungs at necropsy revealed less than $5 \%$ of the area of the lung slice to be emphysematous in two patients, and $25 \%$ in the third.

Further investigations of two of these cases have now been carried out, using large numbers of serial sections to examine the diameters of the bronchioles. In one case (previous patient 3 ) multiple bronchiolar stenoses have been found which we consider to be responsible for the generalized airways obstruction in life.

\section{MATERIALS AND METHODS}

Case I was a 56-year-old man with kyphosis since childhood and a history of progressively severe chronic bronchitis for 16 years. Case II was a 64year-old woman with a history of cough and dyspnoea for 37 years. The clinical histories, radiology, physiological studies, and pathological findings in these cases have already been documented in detail (Simpson et al., 1963). The present cases I and II refer to the previous patients 1 and 3 . Most of the material from patient 2 in that series was unfortunately no longer available for further study.

One lung from each patient was pressure-fixed at the time of necropsy and slices were examined after barium sulphate impregnation (Heard, 1958, 1960). The bronchial tree was dissected, and appropriate blocks were selected for measuring the areas of the bronchial glands. The values were compared with the ranges for normal patients and bronchitics described by Restrepo and Heard (1964). In addition, blocks were subsequently taken from various subpleural regions of the upper and lower lobes in an attempt to include airways with a diameter of less than $1 \mathrm{~mm}$. After secondary fixation in formol-mercury, the blocks were processed by routine methods, embedded in paraffin wax, and cut at 7 microns. Every fifth or tenth section was stained with haematoxylin and eosin, and additional sections were stained for elastic tissue, muscle, and collagen. The resulting material amounted to approximately 1,000 sections containing 40 terminal (non-respiratory) bronchioles, in 34 of which all their branches were examined (respiratory bronchioles and alveolar ducts of the whole acinus). In the remaining six terminal bronchioles, a limited number of branches were available for study. Sixteen bronchioles were from case I and 24 from case II.

\section{RESULTS}

GROSS AND MICROSCOPICAL EXAMINATION OF THE BRONCHIAL TREE

Case I. Some viscid, moderately purulent mucus was present in the large bronchi at the time of necropsy. No firm mucous plugs were seen, although the smaller bronchi contained thin mucus. The bronchial mucosa was unremarkable. There was no dilatation of the bronchi. The area of the bronchial glands in the main right lower lobe bronchus was within the normal limits described by Restrepo and Heard (1964). Examination of a slice of the right lung after pressurefixation demonstrated only a trace of distensive centrilobular emphysema, minimal in degree and involving less than $5 \%$ of the total area of the slice (Fig. 1). The remainder of the slice was normal except for an increase in the anteroposterior diameter due to moderate kyphosis.

The non-respiratory bronchioles examined in serial sections showed no significant microscopic abnormalities. Several foci of pigmented emphysema were present in two of the blocks from the upper lobe, and, when the affected bronchioles could be identified, they were invariably the second-order respiratory bronchioles. An incidental finding was an area of alveolar duct dilatation and alveolar wall thickening in the immediate subpleural region in a block from the lower lobe. There were occasional mild chronic inflammatory infiltrates with bronchiolar scarring, but these were also found in the series from patients without respiratory symptoms. No mucous plugs were seen in the sections. 


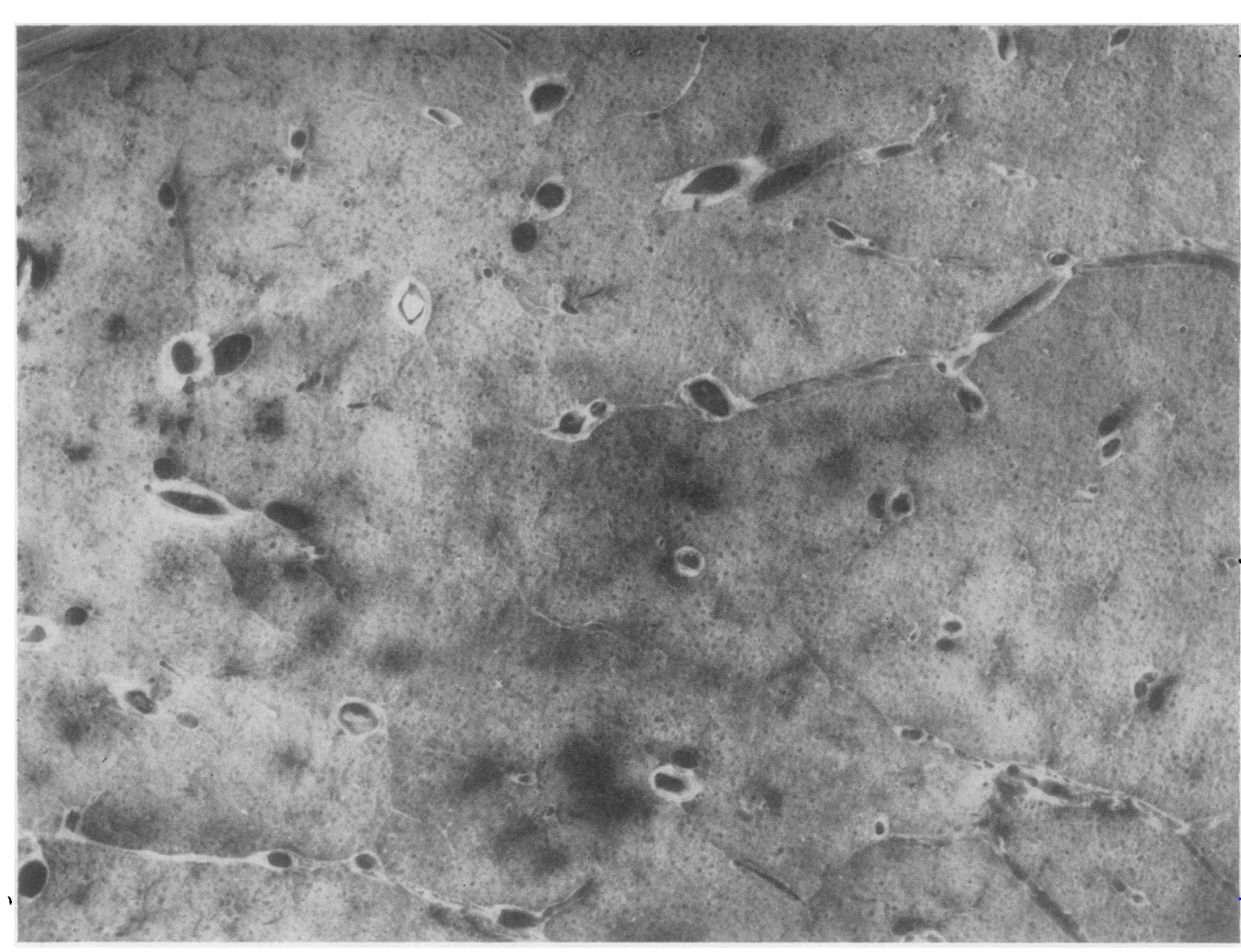

FIG. 1. Case I. Cut surface of the right lung showing normal air spaces in most areas and only a trace of distensive centrilobular emphysema, with pigment. (Pressure-fixation, barium sulphate impregnation, $\times 1 \cdot 8$.)

The scale of the branching confirmed the pattern of spacing described by Reid and Simon (1958). Small bronchi and proximal bronchioles divided at intervals of 1.0 to $0.5 \mathrm{~cm}$. ('centimetre pattern'). In addition to the 'centimetre' and 'millimetre' patterns, we have observed a third pattern in the distal airways. First-order respiratory bronchioles are similar in length and spacing to terminal non-respiratory bronchioles, but the second and third orders are $1 \mathrm{~mm}$. or less in length.

Triple branching was seen, especially at the end of second and third respiratory bronchioles and alveolar ducts, but bifurcation was more common at all levels. Acute right and obtuse angle branching was noted. Of interest was the unequal division seen in non-respiratory bronchioles where the branch with the smaller diameter most often had origin in a right or obtuse angle, and was shorter. The geometry of these variations has been discussed by Weibel (1963). The dimensions of the bronchioles, however, were within the normal limits.

In brief, detailed examination of the bronchi $\stackrel{\text { ? }}{>}$ and bronchioles of this case revealed no structurato changes that would explain the generalized airways obstruction present in life.

Case II (patient 3 in the previous publication) Dissection of the bronchi of both lungs showed $\sigma$ slight dilatation of thin-walled bronchi, especially at the bases. There were occasional peripheraf

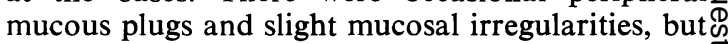
no crypts or other pathological changes were present. The area of the bronchial glands was normal in the main bronchi to both lower lobes $\vec{D}$ and in the left posterior basal bronchus. Inspec- $\frac{?}{\oplus}$ tion of a slice of the left lung revealed a trace $\stackrel{\varrho}{0}$ of distensive centrilobular emphysema and severato small subpleural areas of irregular dilatation and 


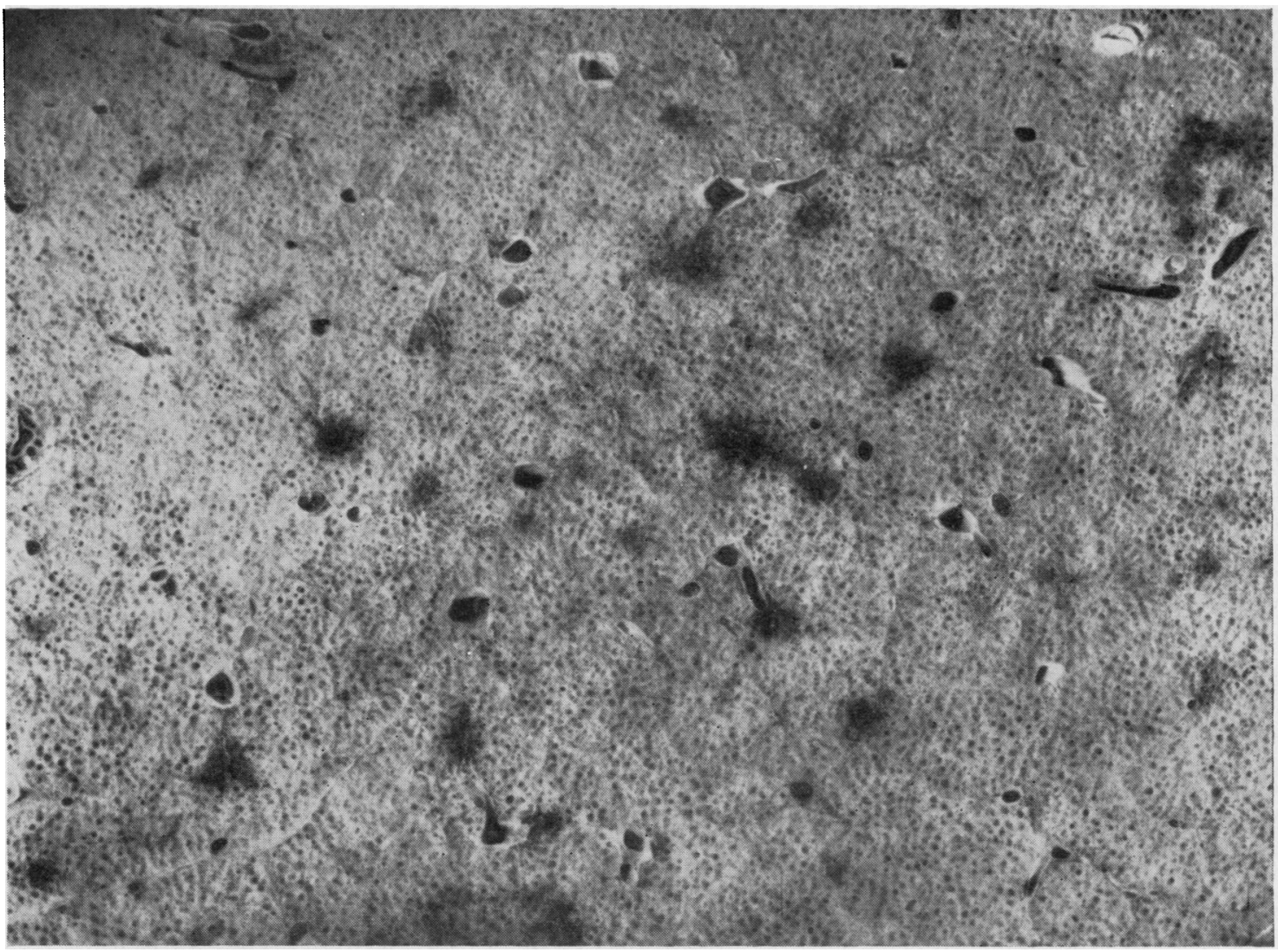

FIG. 2. Case II. Cut surface of the left lung showing normal air spaces in most areas and only a trace of distensive centrilobular emphysema, with pigment. (Pressure-fixation, barium sulphate impregnation, $\times 1 \cdot 8$.)

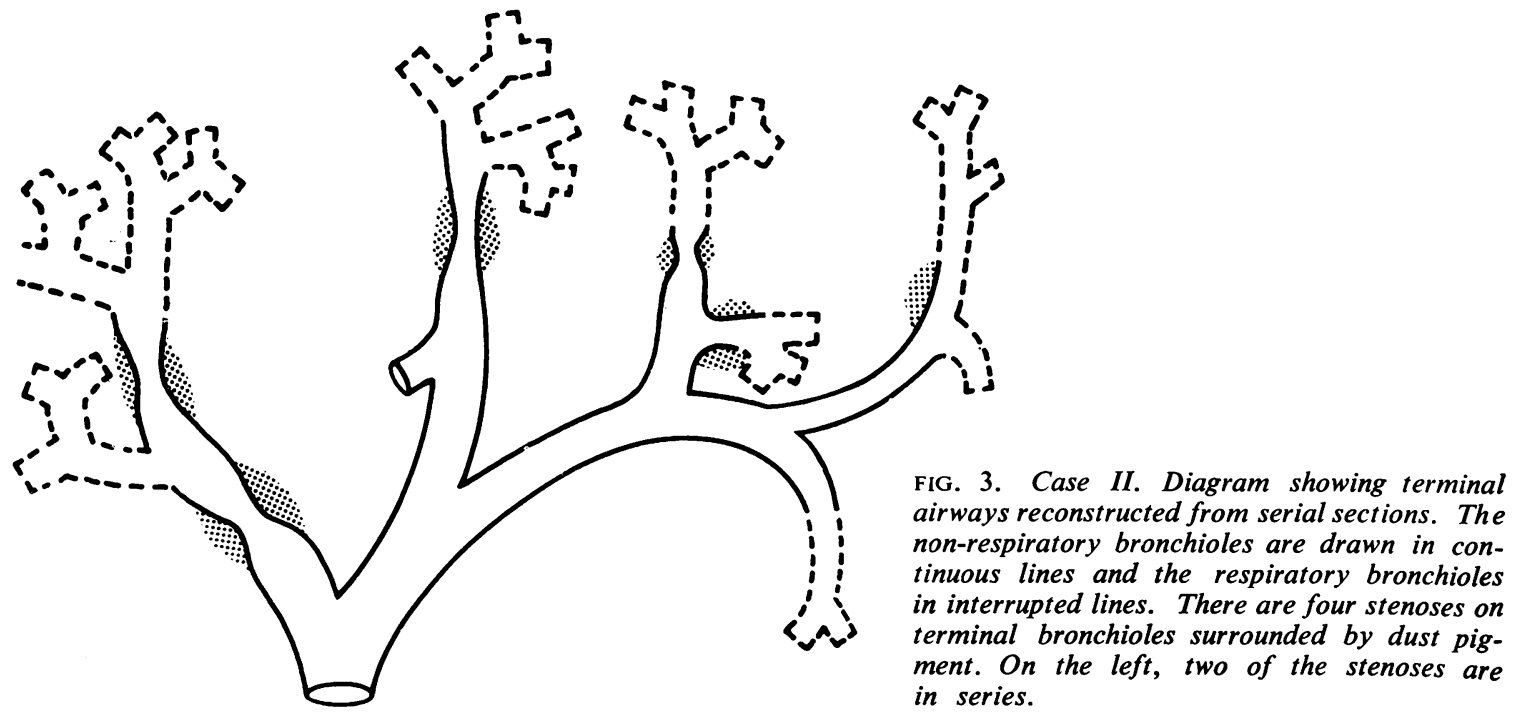

FIG. 3. Case II. Diagram showing terminal airways reconstructed from serial sections. The non-respiratory bronchioles are drawn in continuous lines and the respiratory bronchioles in interrupted lines. There are four stenoses on in series. 


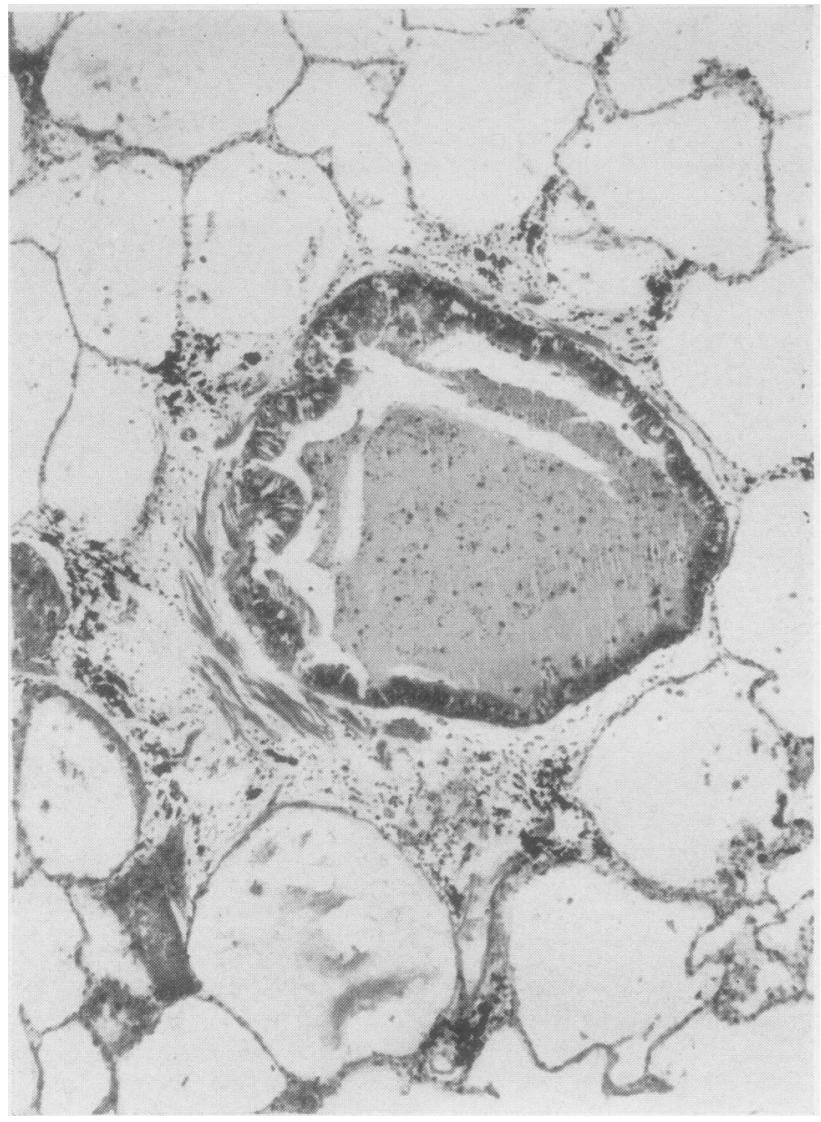

Frg. 4

FIGs 4, 5, and 6. Case II. Samples of serial sections of a non-respiratory bronchiole in order of sectioning. Note the stenosis in Fig. 5 compared with normal diameters proximally and distally in Figs 4 and 6 respectively. (Pressurefixation, haematoxylin and eosin, $\times 63$.)

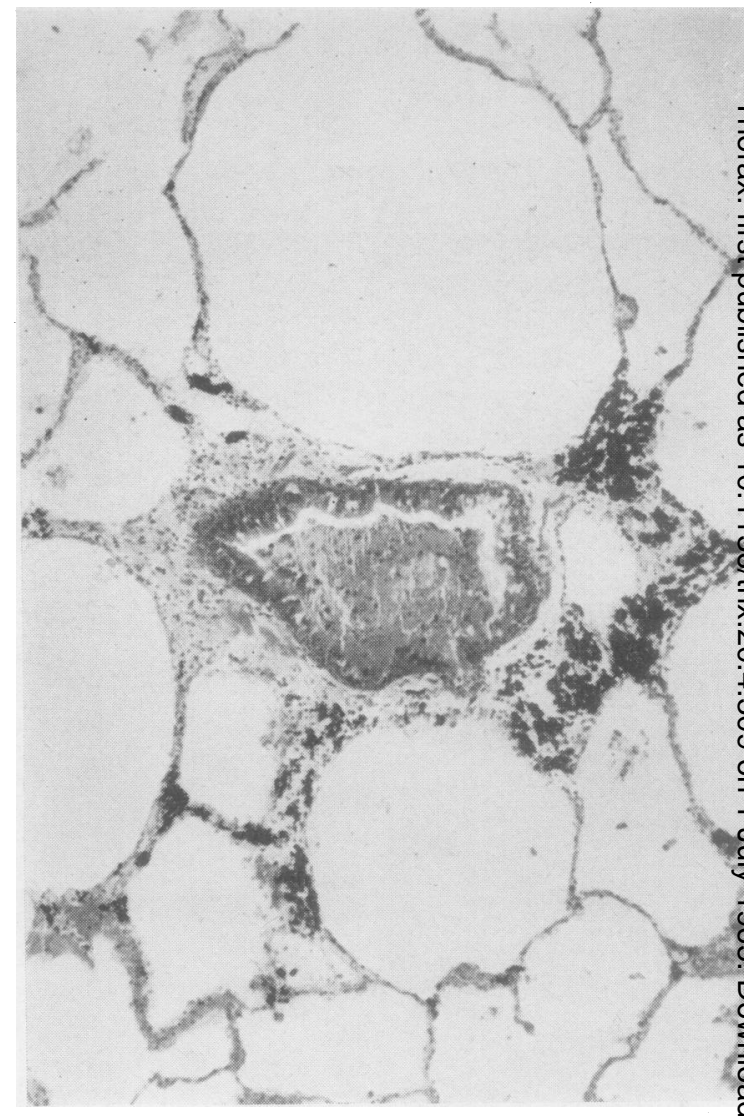

Frg. 5

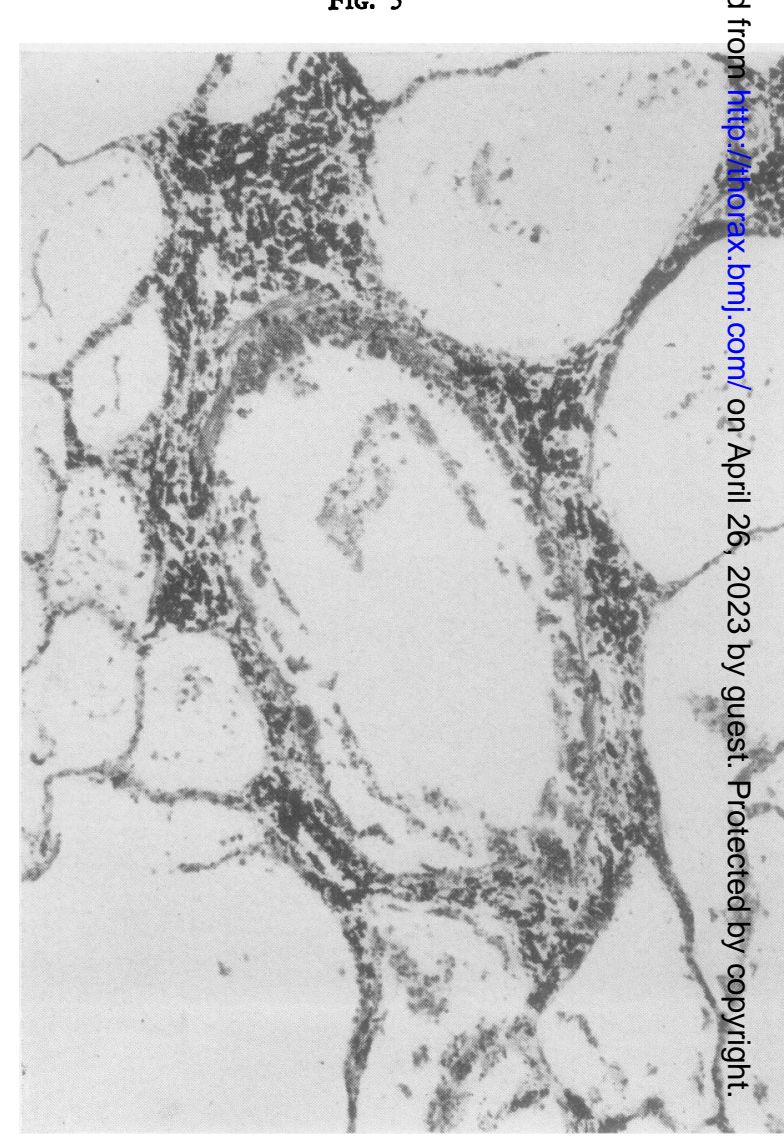

FIG. 6 


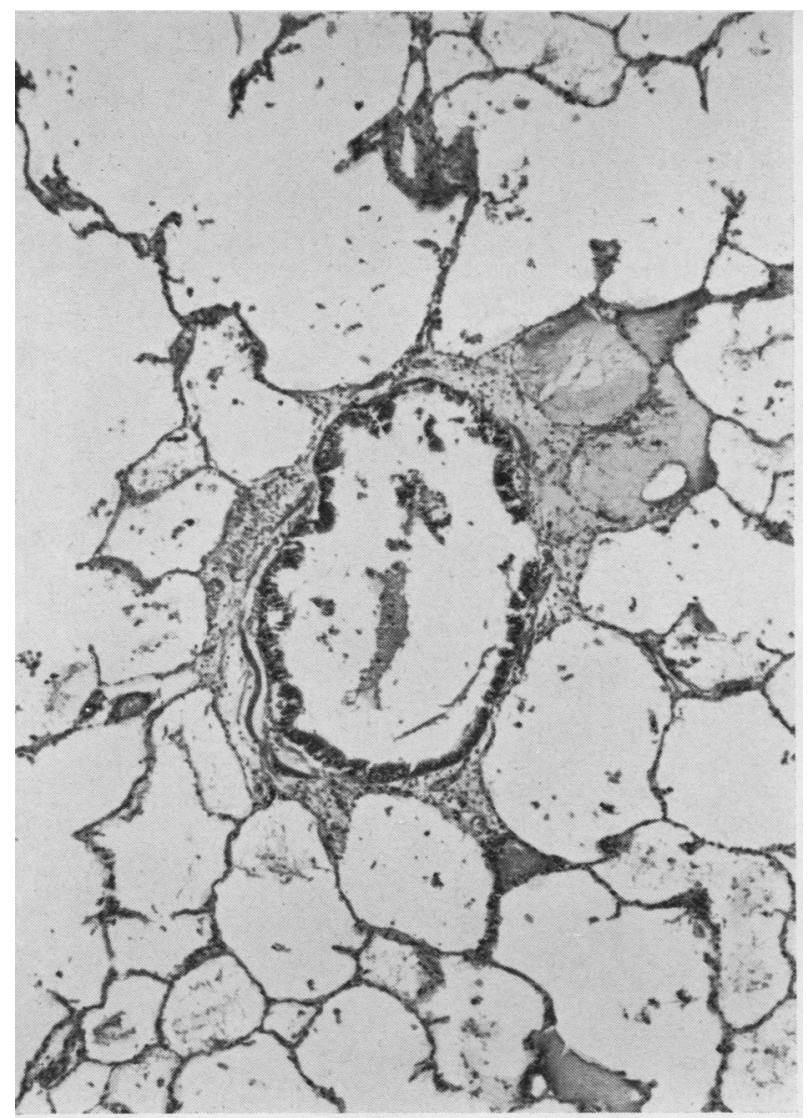

FIG. 7

FIGs 7, 8, and 9. Case II. Samples of serial sections of another non-respiratory bronchiole in order of sectioning. Note the stenosis in Fig. 8 compared with normal diameters proximally and distally in Figs 7 and 9 respectively. (Pressure-fixation, haematoxylin and eosin, $\times 63$.)

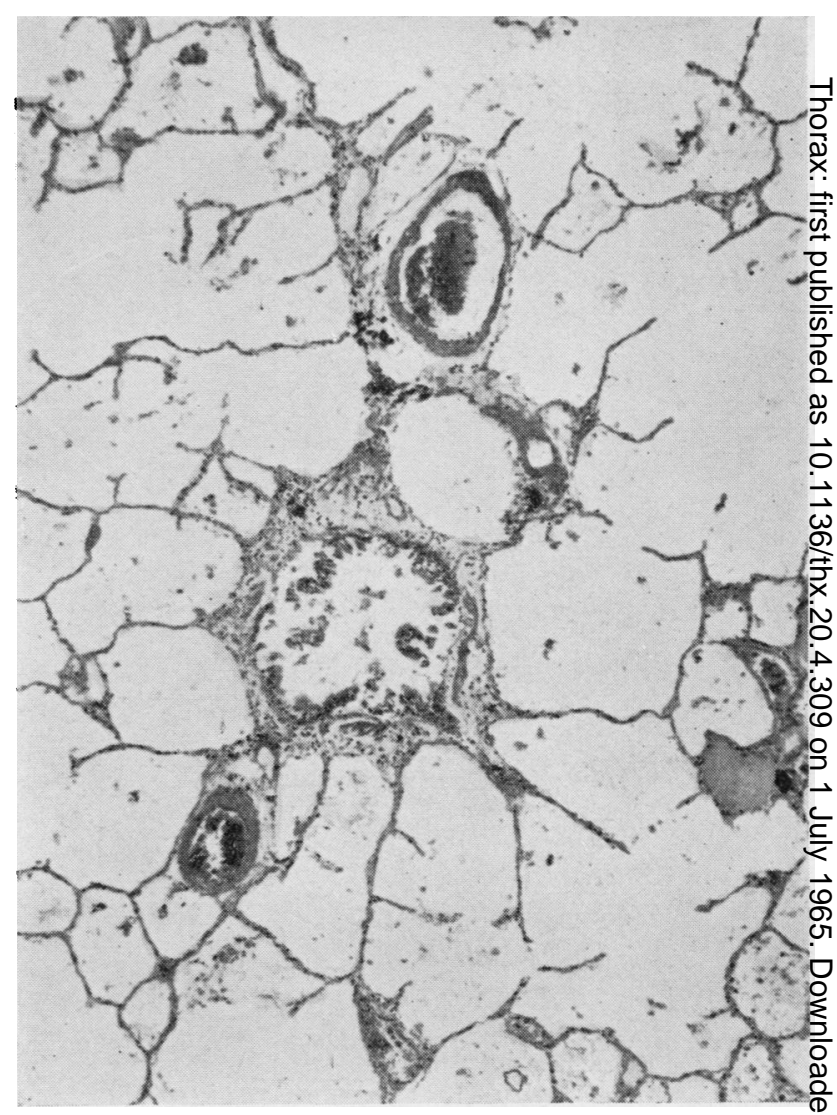

FIG. 8

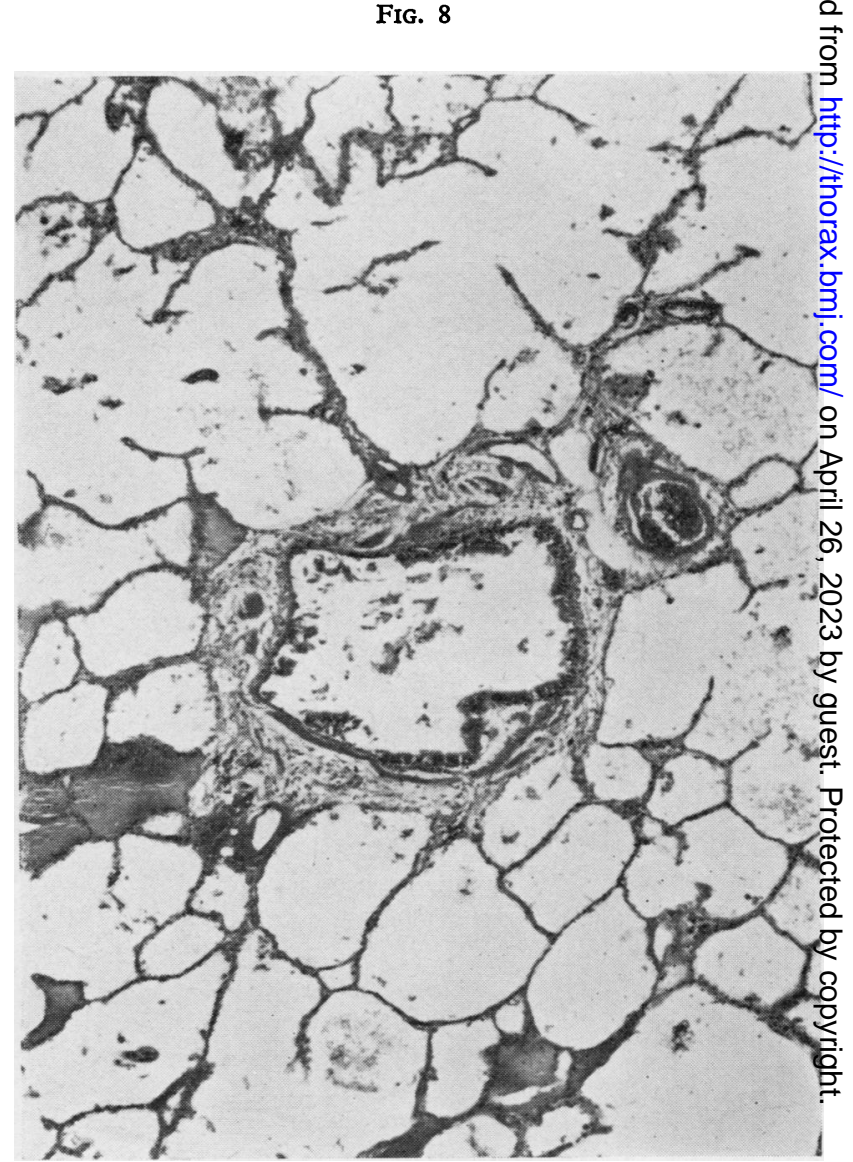

FIG. 9 


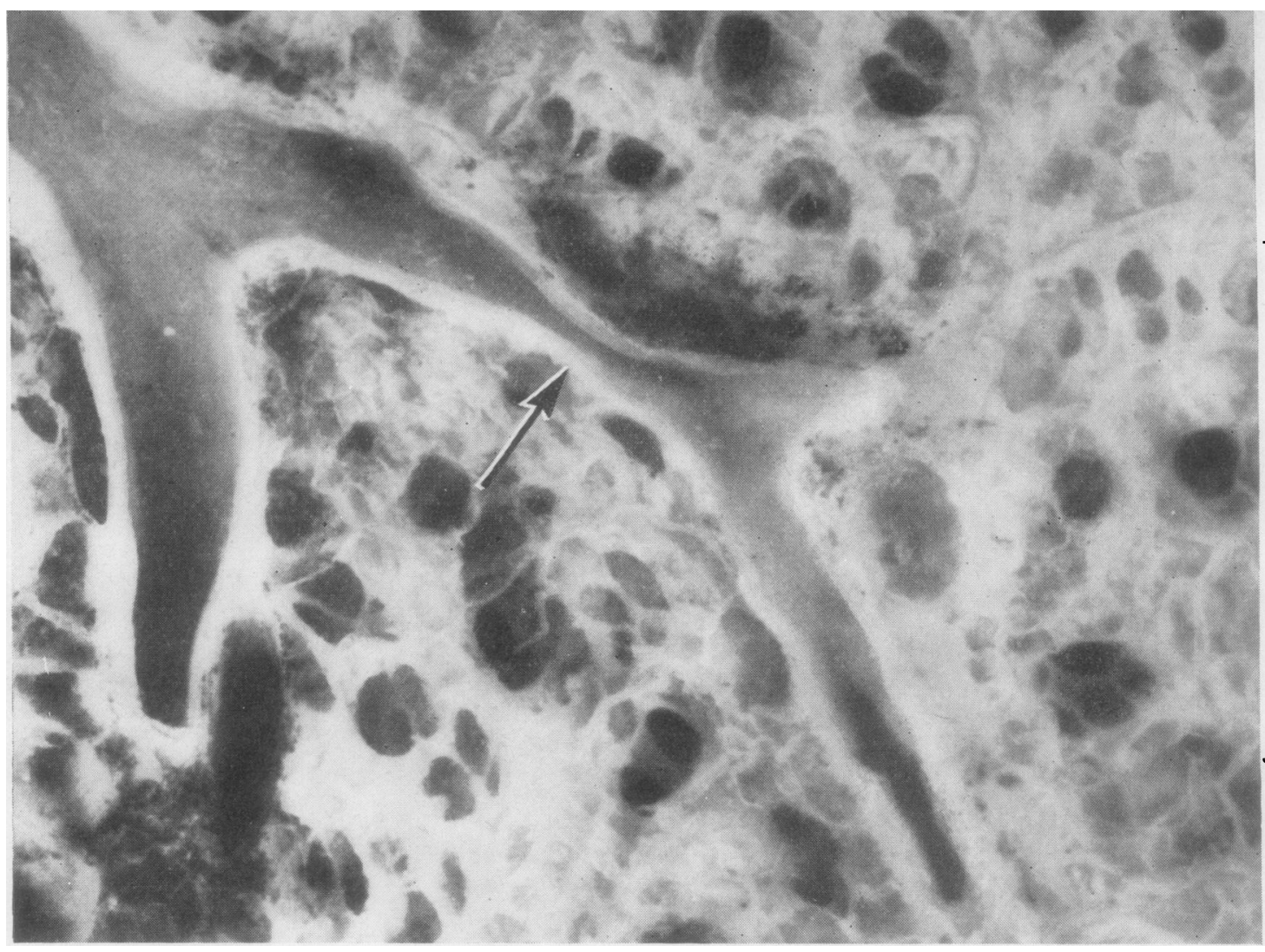

FIG. 10. Case II. Cut surface of the left lung showing probable stenosis of a non-respiratory bronchiole (arrow). (Pressure-fixation, barium sulphate impregnation, $\times 30$.)

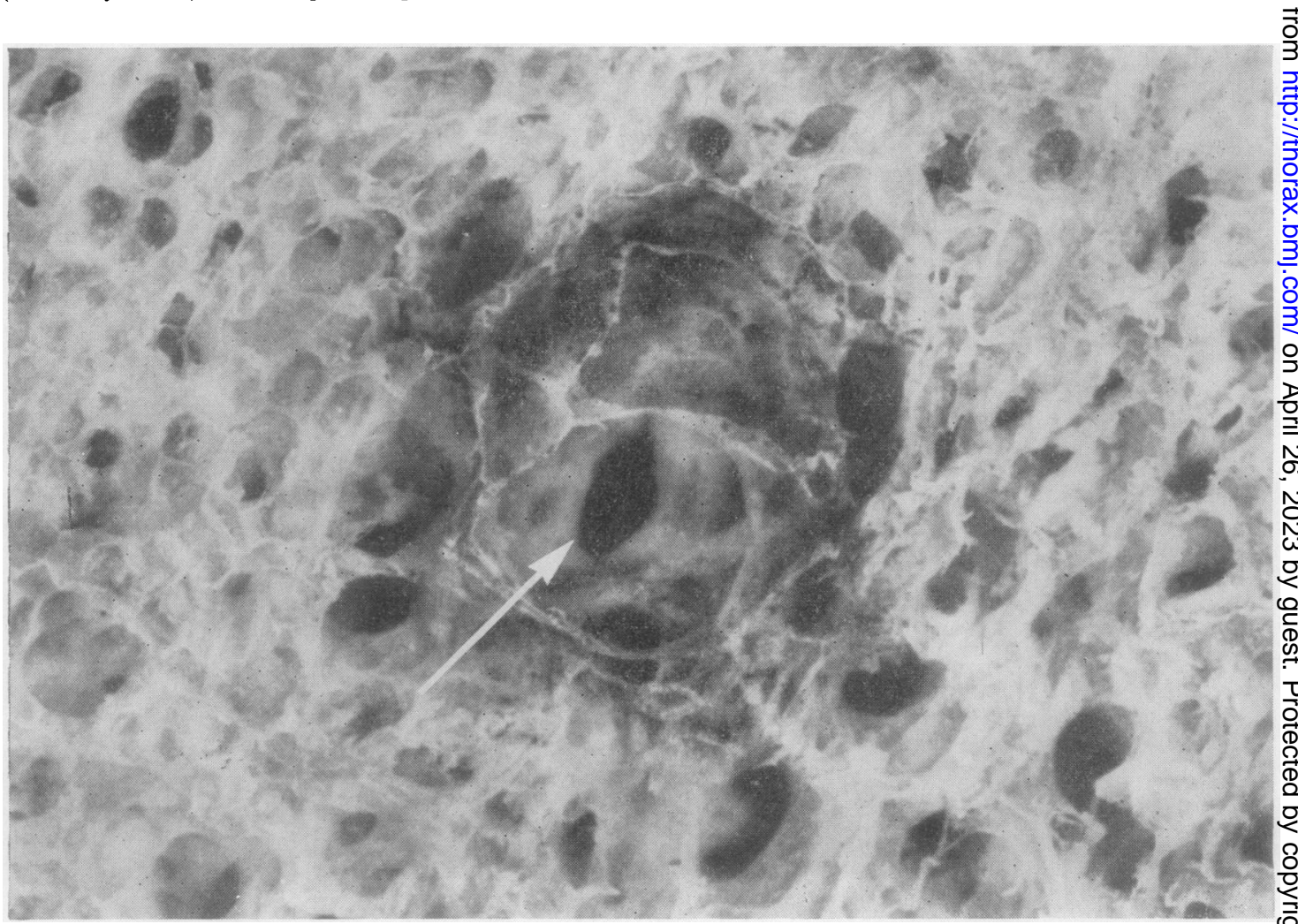

FIG. 11. Case II. Cut surface of the left lung showing probable stenosis of a non-respiratory bronchiole (arrow) $\stackrel{6 \bar{Ð}}{\rightleftharpoons}$ (Pressure-fixation, barium sulphate impregnation, $\times 30$.) 
destruction (Fig. 2). It was estimated that less than $5 \%$ of the total area was involved.

The serial sections of the bronchioles showed several changes. Occasional bronchioles were filled with mucus. Chronic inflammatory cell infiltration, pigmentation, and scarring were mild in degree but widespread. Rare pigmented emphysematous foci involved the second order of respiratory bronchiole. The changes of the greatest interest were stenoses present in the terminal bronchioles as follows.

A total of 24 non-respiratory bronchioles were examined in the serial sections from this case. Seven bronchioles showed areas of stenosis (e.g., Figs 3 to 11). The narrowings were usually 0.3 to $0.4 \mathrm{~mm}$. in diameter, and $0.5 \mathrm{~mm}$. or less in length. The most severe narrowing measured $0.2 \mathrm{~mm}$. in diameter. Proximal and distal to the narrowings, the calibre of the bronchioles was usually normal, i.e., $0.5 \mathrm{~mm}$. in diameter approximately. Two further stenosed airways were interpreted as probable terminal bronchioles. Similar stenoses were seen in three first-order respiratory bronchioles and four proximal non-respiratory bronchioles. Pigmentation and scarring were conspicuous components of the lesions. Moderate infiltrates of chronic inflammatory cells were rarely present. Post-stenotic dilatation was sometimes noted. In one instance two stenoses were found in the same bronchiole (Fig. 3), but elsewhere they were single, though often involving several terminal non-respiratory bronchioles branching from the same proximal bronchioles. Figure 3 is a diagrammatic reconstruction of bronchioles in one set of serial sections. The branching pattern was normal as described above in case $I$ and in the normal controls. The lesions were present in all blocks chosen for serial cutting, and no one portion of the lung appeared to be affected more severely than another. We have seen similar lesions occasionally in serial sections from other cases of severe chronic bronchitis.

The slice of lung was re-examined under water by means of the dissecting microscope with a view to identifying the stenoses initially discovered in serial sections. Two such non-alveolated bronchioles with pigmentation and narrowing were found in the preserved slice (Figs 10 and 11).

We estimate that $45 \%$ to $50 \%$ of the lung substance is supplied by narrowed bronchioles, and they appear to be the principal pathological abnormality in the lungs of case II. In view of the absence of other significant changes we suspect that these narrowed bronchioles may explain the generalized airways obstruction.

\section{DISCUSSION}

Until comparatively recently, emphysema was generally assumed to be the chief cause of respiratory failure in chronic bronchitis. Baldwin, Cournand, and Richards (1949), however, noted that varying anatomical changes may produce similar functional disturbances. A patient who clinically and physiologically presents 'the classical picture of obstructive emphysema, cor pulmonale and right heart failure' may be anatomically one of cor pulmonale in chronic congestive failure with only moderate pulmonary emphysema, or predominantly atrophic pulmonary emphysema with a definite but less striking cor pulmonale. More recently, cases of respiratory failure with minimal emphysema have been reported, such as the three of Simpson et al. (1963), further studied here, and four of Hentel, Longfield, Vincent, Filley, and Mitchell (1963), who used the term 'fatal chronic bronchitis'. Studies by Fletcher, HughJones, McNicol, and Pride (1963), Ogilvie (1959), Simpson (1958) and others have improved the clinical recognition of patients with very little emphysema.

Airways obstruction in chronic bronchitis may be due to a variety of factors, such as accumulating mucus in the bronchi and narrowing of the bronchial lumen by thickened bronchial walls (oedema and congestion of the mucosa and enlarged mucous glands) and by contraction of bronchial smooth muscle, which may also be thickened and hypertrophied. Simon and Galbraith (1953) performed bronchograms on 90 patients with chronic bronchitis and found some abnormality in $79 \%$. The principal abnormalities were excessive variation of the bronchial calibre on respiration, localized beaded bronchial dilatation, bronchial diverticulosis, 'peripheral pooling', and poor filling of the smaller bronchi and bronchioles. However, Reid and Simon (1959) noted that excessive change in the calibre of the large airways during inspiration and expiration, seen in bronchograms, was not common. Many of the changes are reversible. Hentel et al. (1963) found such histological changes as squamous metaplasia of bronchial epithelium, enlargement of bronchial glands, infiltration of the submucosa by lymphocytes, engorged submucosal capillaries and mucocellular plugs, in medium-sized bronchi. They considered the changes suggested that chronic bronchitis was, at least in part, a reversible process, if rational therapy could be started before the onset of hypertensive changes in the pulmonary arterioles. Restrepo and Heard (1964) measured the bronchial cartilage in chronic bronchitis and 
emphysema and found no abnormalities. They concluded that the bronchial collapse, observed radiologically by others and thought by some to be responsible for the increased expiratory flow resistance in emphysema, was not due to degenerative changes in the bronchial wall but to raised pressures in the peribronchial lung, perhaps from narrowing of bronchioles or reduction in their numbers. Hentel et al. (1963) described marked chronic inflammatory changes and fibrosis around bronchioles.

Our present cases showed no abnormalities in the bronchi except some dilatation, and measurements of the bronchial glands were normal, yet the patients died of respiratory failure. Our case I remains unexplained since serial sections of bronchioles have revealed no abnormalities. Either we have yet to find a structural pulmonary lesion of another type in this material or some significance must be attached to the moderate kyphoscoliosis. We are reluctant to accept the latter explanation.

Pure bronchiolar stenosis without emphysema, as in case II, has not been described before as a cause of generalized airways obstruction, but in the absence of other changes we are putting it forward as a suggestion. The lesions were widely distributed throughout the lung and this, together with the absence of other pathological findings in the bronchi or parenchyma, favours such an interpretation. Bronchiolar stenoses have long been appreciated in the presence of emphysema (Spain and Kaufman, 1953 ; Reid, 1954 ; Leopold and Gough, 1957 ; McLean, 1958 ; Anderson and Foraker, 1962).

The term 'bronchiolitis fibrosa obliterans' has been used for generalized airways obstruction after the inhalation of nitrous and other irritating fumes (LaDue, 1941; Darke and Warrack, 1958). We considered the term for case II, but there was no history of exposure to fumes, and the histological appearances were different.

The type of bronchial dilatation seen in case II has only recently been described pathologically in chronic bronchitis (Restrepo and Heard, 1964). The affected bronchi failed to taper as they approached the pleura and the walls were thinned.

\section{SUMMARY}

The lungs from two fatal cases of irreversible $\frac{\bar{c}}{\bar{c}}$ airways obstruction with minimal emphysema $\frac{\widetilde{\sigma}}{\sigma}$ were examined histologically for evidence of $\varrho$ bronchiolar obstruction, using serial sections. Inw one case, multiple bronchiolar stenoses were found $\overrightarrow{0}$ which were considered responsible for the obstruction. The other case remains unexplained.

We wish to thank Mrs. Sally Azzopardi for much secretarial and technical work, Professor C. V. Harri- ̃ son for reading the manuscript, Mr. W. Brackenbury for the photographs, and Mr. D. Banks for Figure 3. One of us (J.R.E.) was in receipt of a grant fromb the National Institutes of Health, and the othero (B. E. H.) a grant from the Medical Research Council.

\section{REFERENCES}

Anderson, A. E., and Foraker, A. G. (1962). Relative dimensions of bronchioles and parenchymal spaces in lungs from norma subjects and emphysematous patients. Amer. J. Med., 32, 218.

Baldwin, E. deF., Cournand, A., and Richards, D. W., Jr. (1949) Pulmonary insufficiency. III. A study of 122 cases of chronics pulmonary emphysema. Medicine (Baltimore), 28, 201.

Darke. C. S., and Warrack, A. J. N. (1958). Bronchiolitis from nitrous fumes. Thorax, 13, 327.

Fletcher, C. M., Hugh-Jones, P., McNicol, M. W.. and Pride, N. B. (1963). The diagnosis of pulmonary emphysema in the presence of chronic bronchitis. Quart. J. Med., N.S., 32, 33.

Heard, B. E. (1958). A pathological study of emphysema of the lungs with chronic bronchitis. Thorax, 13, 136. (1960). Pathology of pulmonary emphysema. Methods of study Amer. Rev. resp. Dis., 82, 792.

Hentel, W., Longfield, A. N., Vincent, T. N., Filley, G. F., and? Mitchell, R. S. (1963). Fatal chronic bronchitis. Ibid. 87, 216.

LaDue, J. S. (1941). Bronchiolitis fibrosa obliterans. Report of a case Arch. intern. Med., 68, 663.

Leopold, J. G., and Gough, J. (1957). The centrilobular form of hypertrophic emphysema and its relation to chronic bronchitis. Thorax, 12, 219.

McLean, K. H. (1958). Bronchiolitis and chronic lung disease. Brit. $J$ Tuberc., 52, 105.

Ogilvie, C. (1959). Patterns of disturbed lung function in patients with chronic obstructive vesicular emphysema. Thorax 14,113

Reid, L. McA. (1954). Pathology of chronic bronchitis. Lancet, 1, 2750 and Simon, G. (1958). The peripheral pattern in the norma bronchogram and its relation to peripheral pulmonary anatomy. Thorax, 13, 103.

- 1959). Pathological findings and radiological changes in chronic bronchitis and emphysema. Brit. J. Radiol., 32, 291.

Restrepo, G., and Heard, B. E. (1964). Air-trapping in chronic bronchitis and emphysema : measurements of the bronchial cartilage. Amer. Rev. resp. Dis., 90, 395. Simon, G., and Galbraith, H.-J. B. (1953). Radiology of chroniç
bronchitis. Lancet, 2, 850.

Simpson, T. (1958). Chronic bronchitis and emphysema. TubercleN (Lond.), 39, 307.

Heard, B. E., and Laws, J. W. (1963). Severe irreversible airways obstruction without emphysema. Thorax, 18, 361.

Spain, D. M., and Kaufman, G. (1953). The basic lesion in chronico pulmonary emphysema. Amer. Rev. Tuberc., 68, 24.

Weibel, E. R. (1963). Morphometry of the Human Lung, p. 1140 Springer, Berlin. 